\title{
A New Kinetic Model of a Growing Bacterial Population
}

\author{
By C. J. PERRET* \\ Guinness-Lister Unit, Lister Institute of Preventive Medicine, Chelsea Bridge Road, \\ London, S.W. 1
}

\begin{abstract}
SUMMARY : A chemical open system of fixed volume in a constant environment tends towards a steady state in which its mass remains unchanged. Such a system is not a satisfactory kinetic model of a growing bacterial population, which increases its mass and volume, or grows, logarithmically, in a constant environment. However, when the material limiting the volume of an open system is itself one of the dynamic components the system can then grow logarithmically of its own accord. If the surface-area-to-volume ratio of such an 'expanding system' remains unchanged logarithmic growth can continue indefinitely, and in a constant environment the system enters a time-dependent 'exponential state'. Autocatalysis is not involved in the logarithmic growth of an expanding system; but when an autocatalytic stage is included the growth curve can exhibit a typical log-phase during which growth rate is virtually independent of concentrations of source material above a threshold level. The properties of expanding systems are deduced from those of open systems by non-mathematical arguments, and some of the implications of the expanding system concept in practical and theoretical microbiology are discussed. It is suggested that the spontaneous occurrence of expanding systems in a non-living environment might be the first step towards the evolution of living organisms.
\end{abstract}

\section{OPEN SYSTEMS}

The foundations of the general theory of open systems have been presented, discussed, and some aspects analysed in detail, by Burton (1936, 1939), Reiner \& Spiegelman (1944), Spiegelman \& Reiner (1945), Hinshelwood (1946, 1952, 1953 $a, b)$, Denbigh, Hicks \& Page (1948), Denbigh (1951, 1952) and Dean \& Hinshelwood (1955) in papers which provide references to the works of many other authors. Specific applications of the theory to continuous chemical reactions and continuous culture have also been published by Denbigh (1944, 1947), Monod (1950), Novick \& Szilard (1950), Danckwerts (1954), Denbigh \& Page (1954), Spicer (1955), Herbert, Elsworth \& Telling (1956), Perret (1956, 1957) and Moser (1957). The initial section of this paper starts with a non-mathematical description of the properties of simple open systems, based on the publications cited above (particularly those of Burton, 1939 and Denbigh et al. 1948). The discussions of compound and autocatalysed open systems and of expanding systems are not based on previous publications.

Throughout the paper all reactions are treated as reversible, since there seems to be no justification for the concept of an irreversible chemical change. The reactions are also assumed to be homogeneous, unimolecular, and of the first order, except where otherwise stated. Those assumptions are made in order to keep the examples in the text as simple as possible; but the

* Present address : Department of Microbiology, University of Western Australia, Perth, Western Australia. 
conclusions reached seem equally valid in principle for systems involving heterogeneous reactions, and for multimolecular reactions of any order higher than zero. Consideration of the effects of pressure, irradiation intensity and temperature are outside the scope of this article; those parameters are therefore assumed to remain unchanged throughout the discussion.

\section{Simple linear open systems}

Since the behaviour of an open system depends on its chemical and physical attributes, those which are relevant must be defined. The initial arguments deal with hypothetical idealized systems, so it is unnecessary to complicate the issue at this stage by considering to what extent, or under which circumstances, their attributes could be realized in practice.

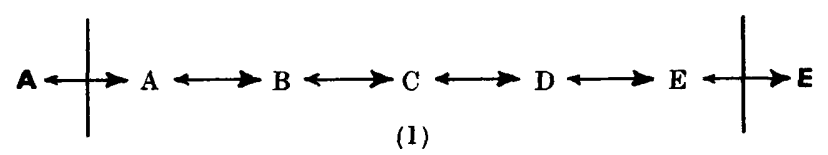

In Ex. 1 the symbols $\mathbf{A}$ and $\mathbf{E}$ represent respectively the source and sink materials, which are assumed to be in homogeneous solution, at fixed concentrations, in the unlimited 'chemical environment' or 'medium' surrounding the system. The concentrations of the source and sink materials are further assumed to be respectively above and below those levels at which the compounds would be in equilibrium with each other; for most purposes, the concentration of the sink materials may be taken as virtually zero. In the steady state there is, therefore, a 'flux' of material through the system from left to right, as indicated by the thickened arrow-heads. The system itself is not unlimited, but has a fixed volume, being enclosed or delimited by a 'boundary layer' represented by the vertical lines. That boundary layer (which may be imagined as a membrane or interface) has the special property of being permeable to the source and sink materials, $A$ and E, but completely impermeable to the intermediate compounds $\mathrm{B}, \mathrm{C}$ and $\mathrm{D}$. The materials inside the system are in homogeneous solution, in a solvent which may, or may not, be identical with that of the medium.

The limitations of two-dimensional symbolism prevent Ex. 1 from giving a realistic picture of the state of affairs. Since the same conventions will be used repeatedly in subsequent expressions, it may be as well to emphasize here the way in which they are misleading. The representation of source and sink on opposite sides of the system does not mean that they are necessarily physically separated. The medium surrounding the system on all sides can be a single entity serving the dual function of both source and sink; and throughout this paper it will be assumed that such is the case. Furthermore, the arrangement of symbols inside the system represents a sequence of events in 'time', not 'space'. Thus, I describe $\mathbf{A}$ and $\mathbf{B}$ as 'kinetically proximate' because a molecule of $A$ is much more likely to become a molecule of $B$ than anything else: $\mathbf{A}$ and $\mathrm{E}$, on the other hand, are 'kinetically remote' because a molecule of $\mathbf{A}$ is unlikely to become $\mathbf{E}$ without first passing through the inter- 
mediate forms B, C and D. Nevertheless, the homogeneity of the system implies that molecules of $\mathbf{A}$ and $\mathbf{E}$ will often be immediately adjacent in space: and kinetic theory requires the assumption that $\mathbf{A}, \mathbf{B}, \mathrm{C}, \mathrm{D}$ and $\mathbf{E}$ are all sometimes transformed into each other directly. But if such direct transformations are not indicated by arrows their rates are assumed to be negligibly minute. The thickened arrow-heads indicating the direction of flux through the system in Ex. 1 therefore show the most probable order in which a group of atoms will assume the various molecular forms; I call that order the 'dominant sequence' of reactions or transformations linking $\mathbf{A}$ and E. The linear sequence of symbols does not therefore imply a regular spatial migration of material from one side of the system to the other, or exclude the existence of potential alternative pathways which may in turn become significant or even dominant under altered conditions of temperature, pressure, radiation intensity or catalysis.

In a constant environment all simple linear open systems, regardless of their length, tend towards a steady state in which the concentrations of all molecular species remain constant indefinitely, except for fluctuations resulting from the random nature of the events governing rates of chemical change. The steady-state concentration of each of the molecular species within the system does not depend on its initial concentration, but depends only on the concentrations of source and sink materials, and on the velocity constants of every stage in the transformation sequence. The term 'equifinality,' proposed by Bertalanffy (Burton, 1939), is sometimes used to describe the independence of the initial and steady-state concentrations of molecular species in the system.

\section{Transition between steady states in simple linear open systems}

An open system of fixed volume contains, in the steady state, a constant mass of material; for if the concentration, and therefore the amount, of every individual molecular species is time-independent, then the sum of the masses of all the species must also be time-independent. In contrast, an open system which has not yet reached a steady state will generally have more material entering than leaving, or vice versa; it will then either increase or decrease in mass.

During transience towards a steady state some of the components of an open system can exhibit 'overshoot', 'false start' and 'false equilibrium', if suitable assumptions are made about the initial and final parameters. The resemblance between those transient phenomena and some aspects of the behaviour of living organisms has been commonly misinterpreted as evidence for regarding organisms as open systems. However, such time-dependent changes cannot, by definition, occur in open systems in the steady state, or in closed systems in equilibrium; but they are equally probable in both open and closed systems during transience towards time-independence. In fact, an open system may be regarded as no more than a portion of a closed system which is, as a whole, in transience towards an equilibrium imperceptibly approached during the period of observation. The sole and sufficient reason 
for regarding organisms as open systems is that they exchange material with their environment.

A type of transience particularly pertinent to the subsequent discussion in this paper is that produced by a change in the composition of the medium in which an open system has reached a steady state. To avoid confusion, the resulting special form of transient behaviour is called 'transition'. Returning to the linear open system of Ex. 1, let it be assumed that, after the system has reached a steady state, the concentration of $\mathbf{A}$ in the medium is instantaneously doubled. As a result of the change, the components of the system will all be present at less than their steady-state concentrations for the new medium. Extra A will then move into the system, and all the concentrations will rise towards their new levels; the system will increase in mass. The extra material can only be gained by inward diffusion from the medium; and since rate of diffusion is proportional to concentration gradient, the rate of increase of mass will be greatest immediately after the change, becoming progressively slower as the new steady state is approached. The concentrations of the various components of the system will be affected successively in the order of their kinetic distance from the source, and the transition will take the form of a surge of increased concentration sweeping through the dominant sequence from source to sink. If, on the other hand, the concentration of the sink material were raised while that of the source remained constant, the surge would travel in the reverse direction; and if both concentrations were raised the surges would travel in from both ends towards the centre. Lowered concentrations of source or sink would lead to negative surges. In general, from any parameter of the system which is altered, the effect spreads out to successively more and more kinetically remote components.

The usual mathematical expressions for the curve of transition from one steady state to another imply that the change is complete only after infinite time. In fact, that is not strictly true for a system containing a limited number of molecules: after a finite time the various concentrations are so near to the steady-state level that their deviation merges into the general background of statistical fluctuations; the state is then as steady as it will ever be.

\section{Concentration gradients in open systems}

There can be a flux through an open system only if there is some form of gradient from source to sink. In chemical open systems that gradient exists by virtue of the disparity between the concentrations of source and sink materials, which are respectively above and below those which would obtain at equilibrium. But if the equilibrium constant for the transformation $A \leftrightarrow E$ is greater than unity, then the molar concentration of the sink material $E$ may exceed the molar concentration of the source material A. Use of molar concentrations can therefore conceal from casual inspection the existence of a gradient, or give a misleading impression of the direction of flux. In a long dominant sequence where the equilibrium constants of some reactions are much less than unity and of others much more than unity, it becomes virtually impossible to 
envisage the state of affairs within the system from a consideration of molar concentrations. That difficulty can be overcome in the case of a sequence of unimolecular first-order reactions by using, for each component, the ratio of its molar concentration within the system to its molar concentration when in equilibrium with any fixed arbitrary concentration of the source material. I call the resulting dimensionless value the 'flux potential' of the component. For reactions of higher order than the first the calculation of flux potentials is, of course, more complex, since it involves the use of powers or products of concentrations: but such cases do not concern us here.

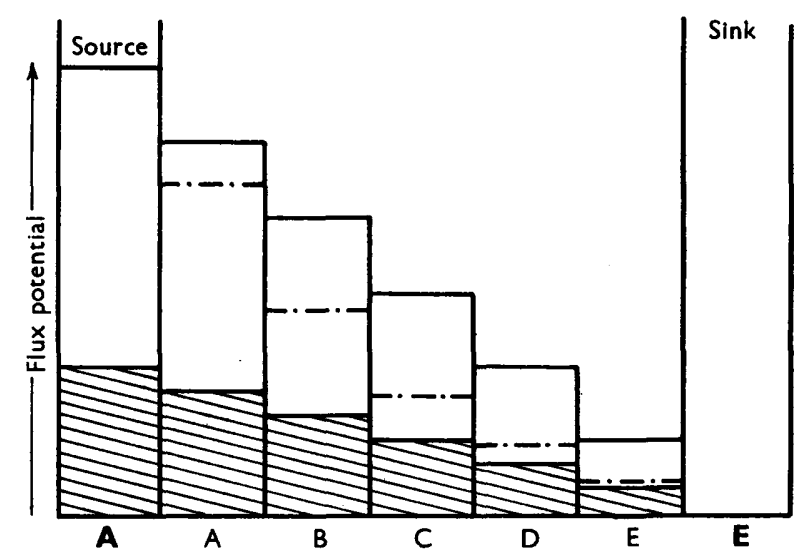

Fig. 1. The effect of trebling the concentration of suurce material on the flux potentials of components of a hypothetical simple linear open system. The concentration of sink material is shown as zero for the sake of clarity. The hatched and unhatched columns represent respectively the initial and final steady states. The broken lines show how the gradient of flux potentials is steeper during transition than during either of the steady states.

When concentrations are expressed as flux potentials all equilibrium constants assume a value of unity, because the flux potentials of all components are equal at equilibrium. If the flux potentials of the components of an open system do not decrease progressively from source to sink, there can be no flux through the system (though there may be fluxes into and out of it); and it cannot be in a steady state. If the flux potentials do decrease progressively there must be a flux through the system, and it may be in a steady state; but need not be so. Figure 1 depicts in terms of flux potentials the state of affairs before, during and after the type of transition considered in the previous section. That the gradient of flux potentials is steeper during transition than in either the initial or final steady states is pertinent to the properties of the expanding systems considered later.

\section{Simple reticular open systems}

The representation of an open system used in Ex. 1 implies that there is a negligible flux through each of the inevitable alternative pathways; that is, that the system is linear. When alternative pathways contribute significantly 
to the total flux the system may be described as 'reticular'. A system may be reticular by virtue of 'closed loops' if the alternative pathways both originate and terminate within the system; or by virtue of 'open loops' if more than one type of substance forms the source or sink material.

Let it be assumed that the source contains, in addition to $A$, another substance $\mathbf{M}$, linked by a transformation sequence to an additional sink substance, Q.

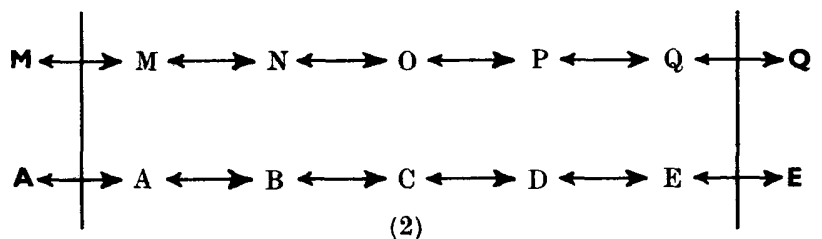

As it stands, Ex. 2 obviously represents two completely independent simple linear open systems in which the flux from $\mathbf{M}$ to $\mathbf{Q}$ will have no effect on the flux from $\mathbf{A}$ to $\mathbf{E}$. The steady state of either sequence can be modified without altering the other, and the two sequences are completely 'uncoupled'.

If, however, it is assumed that the two components $\mathrm{O}$ and $\mathrm{C}$ can be formed from each other, the situation changes. Clearly, an alteration in the concentration of $O$ will then affect the concentration of $C$, and vice versa. The two

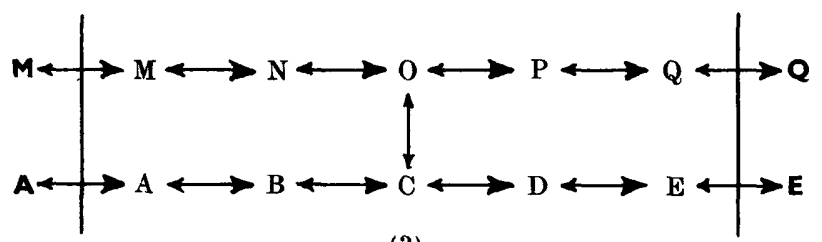

(3)

sequences are now 'coupled', and the extent of their coupling depends on the magnitude of velocity constants for the $\mathrm{O}, \mathrm{C}$ transformation: as they tend towards zero the coupling will become 'looser' until, at the limit, the sequences are uncoupled. On the other hand, as the velocity constants tend towards infinity the coupling will become 'tighter' until, at the limit, $\mathrm{O}$ and $\mathrm{C}$ are identical species and the sequences are 'fully coupled', as shown in Ex. 4.

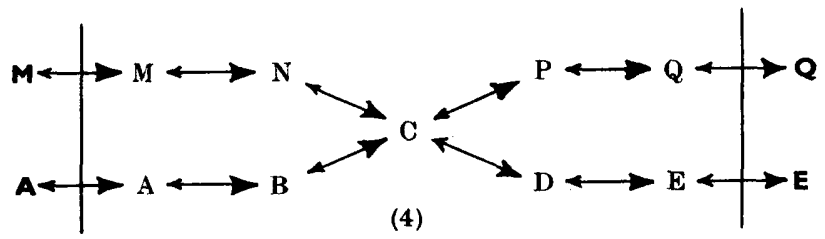

Numerous other modifications are possible: for instance, omission of the components $\mathbf{M}, \mathbf{N}$ or $\mathbf{P}, \mathbf{Q}$ would produce convergent or divergent sequences. In each case, the extent to which the flux through one portion of the system modified the flux through other parts would depend on the relative magnitude of the relevant velocity constants; that is, on the degree of coupling. 


\section{COMPOUND SYSTEMS}

Linear or reticular open systems of any degree of complexity will always tend towards a steady state. That state will eventually be attained in a constant environment only if all velocity constants assume unchanging values and if all open loops originate or terminate outside the system. If velocity constants and concentrations have interdependent values a reticular system may enter a state in which concentrations permanently oscillate about a mean value. Such behaviour is familiar in the fields of electronics and servo-mechanisms: the classical example in biology is provided by the predator-prey relationship (Volterra, 1931). However, the conditions for stable oscillation are rather exacting, and there are other, more likely, circumstances under which an open system in a constant environment may fail to attain a steady state.

\section{Internal sinks and internal sources}

Let it be assumed that a system of the type previously shown in Ex. 1 is set up in a liquid medium with $\mathbf{A}$ and $\mathbf{E}$ in solution; and that before a steady state is reached, the solubility of component $D$ in the system is exceeded

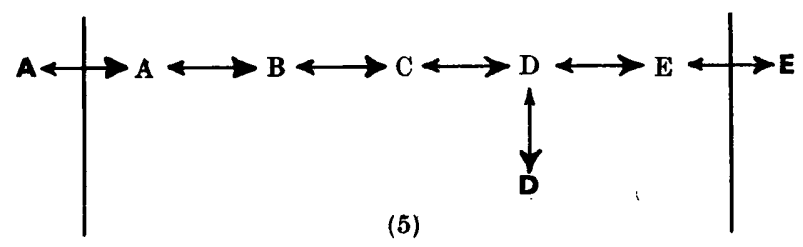

Under these circumstances $D$ will remain at a concentration which is constant, but below its steady-state level. The flux through the sequence A, B, C will be greater than the flux through $\mathbf{E}$ by the amount of $\mathbf{D}$ thrown out of solution in unit time. Further increases in the concentration of $\mathbf{A}$ will raise the concentration of $\mathbf{A}, \mathbf{B}$, and $\mathbf{C}$; but will not affect the concentrations of $\mathbf{D}$ and $\mathbf{E}$. The insoluble component $D$ therefore becomes an 'internal sink' for the sequence $A, B, C, D$ and an 'internal source' for the sequence $D, E$. Since the system now consists of two partially independent open sequences it may be called a 'compound open system'. In this case the system will have a limited 'life' because the accumulation of the solid phase D will lead to its extinction.

A similar effect can be obtained by taking off from the main sequence any side-branch forming an open loop which terminates inside the system instead of in the medium (Ex. 6). For instance, if $D$ can be transformed not only to $\mathrm{E}$, but also to $Y$ via $X$, and if the membrane bounding the system is as impermeable to $\mathrm{X}$ and $\mathrm{Y}$ as it is to $\mathrm{B}, \mathrm{C}$ and $\mathrm{D}$, then the system will again become compound, because the side branch forms, in effect, a closed system tending towards false equilibrium. But in this case the compound system would not be selfextinguishing because $\mathrm{Y}$ would not accumulate indefinitely. If the reactions in the side branch have rates of the same order as those in the main sequence, then the system will reach its compound steady-state and false equilibrium 
condition in one smooth sequence. On the other hand, if one of the side branch reactions, such as $\mathrm{D} \longleftrightarrow \mathbf{X}$, is very slow, then the main sequence will first reach what is almost a steady state, while a small proportion of $D$ is continually diverted to build up the $X, Y$, sequence towards an equilibrium which may not be attained for a very long time. If $Y$ is not sufficiently soluble to rise to a concentration in false equilibrium with the steady-state concentration of $\mathrm{D}$, it will be precipitated; the system will then become self-extinguishing.

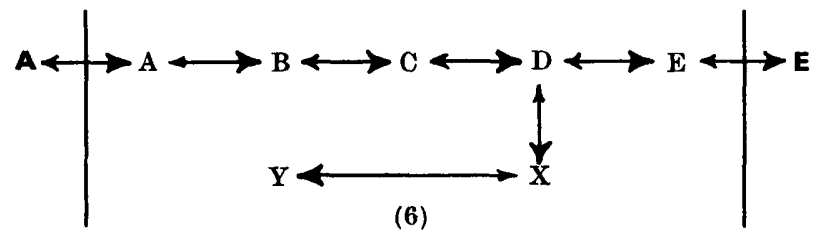

Compound systems due to catalysis

A compound open system can also arise if one or more of the transformation stages is suitably catalysed. Let the system in Ex. 7 be set up in a low concentration of $\mathbf{A}$ and contain a fixed excess of an extraneous catalyst $b c$ for the stage $B \leftrightarrow C$. The catalyst will have the effect of greatly increasing

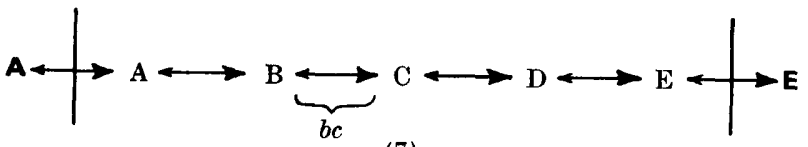

(7)

$\mathrm{k}_{\mathrm{BC}}$ and $\mathrm{k}_{\mathrm{CB}}$, but the reaction will still be, in effect, of the first order. The system will reach a steady state. If the concentration of $\mathbf{A}$ is now progressively increased, the concentrations of each of the components of the system, A, B, $\mathbf{C}, \mathbf{D}, \mathbf{E}$ will initially rise in the normal way. However, since the concentration of $b c$ is constant, the ratio $b c / \mathbf{B}$ will diminish as the concentration of $\mathbf{B}$ increases, so that eventually $b c$ will no longer be present in excess. Finally the system may reach a stage in which so much $B$ is present that the catalyst is permanently 'saturated'; the reaction $B \leftrightarrow C$ will then virtually be of 'zero order' with respect to $B$, since it is occurring at a constant rate, proportional to the amount of $b c$, but independent of the concentration of $\mathrm{B}$ itself. C then becomes an internal source for the sequence $D, E, E$, because further increases in the concentration of $\mathbf{A}$ will raise the concentrations of $\mathbf{A}$ and $\mathbf{B}$; but will have very little effect on the concentrations of $\mathbf{C}, \mathbf{D}$ and $\mathbf{E}$ if, in the absence of the catalyst, $\mathrm{k}_{\mathrm{BO}}$ is so minute that the uncatalysed reaction contributes a negligible proportion of the total $\mathbf{C}$ produced. The flux through the system can therefore be virtually independent of concentrations of $\mathbf{A}$ above some minimum value.

In this case $\mathbf{B}$ will not act as an internal sink for the sequence $\mathbf{A}, \mathbf{A}, \mathbf{B}$ : on the contrary, for very high concentrations of $A$, the flux through $B$ will eventually be so small a fraction of the total $B$ present that the $A, A, B$ sequence will tend towards false equilibrium. 


\section{Autocatalysis in compound systems}

In living systems the catalysts are not of extraneous origin, but are themselves produced in the system, constituting components which must be included in the transformation sequence. It is interesting to consider the effect of such 'autocatalysis' in an open system.

Let it be assumed that substance $Y$, which terminates the side-branch in Ex. 6, catalyses the reaction $B \leftrightarrow C$ by being, or becoming, $b c$ : and that the system is set up, but has not yet reached the steady state, in a medium containing a high concentration of $\mathbf{A}$.

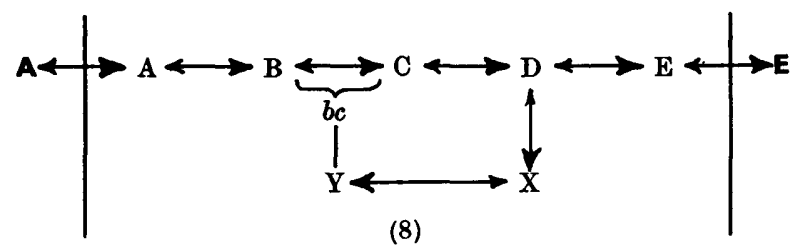

As the main sequence moves towards a steady state the concentration of $b c$ will rise, the rate of the reaction $B \leftrightarrow C$ will thereby increase, and the concentration of $b c$ will rise yet higher. That autocatalytic cycle will not, however, continue indefinitely; the maximum rate at which $B$ can be supplied is fixed by the stages $\mathbf{A} \leftrightarrow \mathbf{A}$ and $\mathbf{A} \leftrightarrow \mathbf{B}$ which, for a fixed concentration of $\mathbf{A}$, cannot exceed the limiting speed which they would attain if the concentration of $B$ were zero. The main sequence will therefore approach a steady state in which the ratio of the concentrations of $B$ and $C$ is virtually that of equilibrium, because $B \leftrightarrow C$ will be a very fast stage enclosed by two relatively slow stages, $\mathbf{A} \leftrightarrow \mathbf{B}$ and $\mathbf{C} \leftrightarrow \mathbf{D}$. The side branch will approach false equilibrium if $\mathbf{Y}$ is sufficiently soluble: otherwise it also will tend towards a steady state, with a flux from the source $\mathbf{D}$ into the sink of insoluble $\mathbf{Y}$. In the case of autocatalysis, unlike that of extraneous catalysis, the flux through the system can never be virtually independent of the concentration of $\mathbf{A}$ until the solubility of one of the components of the system is exceeded.

\section{The open system as a model bacterial population}

During recent years it has become increasingly fashionable to invoke open systems and the steady state when discussing aspects of bacterial growth and metabolism. The general attitude seems to be that a growing bacterial population has the attributes of the simple linear sequence shown in Ex. 1, but is at the same time very much more complicated. The exact nature of the complications is usually left vague; but when specified they are often incompatible with the properties of a simple open system, and the interpretation of the complex in terms of the simple becomes invalid. The result is a most unsatisfactory state of ambivalence, whereby the open system is cited, often wrongly, in justification of any viewpoint which it seems to support; but dismissed as inadequate whenever its presumed properties apparently conflict with those of the living system being studied. It may therefore be as well, at this stage, to 
consider the extent to which an open system can manifest the phenomena associated with the growth and metabolism of a bacterial population. For this purpose the entire bacterial population within a culture may be regarded as a single system, the 'biophase', having a constant surface-area-to-volume ratio.

A simple linear open system and the biophase of a bacterial culture have in common the property of continuously taking in from the medium source material (nutrients) which, after a series of chemical transformations (metabolism), are excreted as sink material (waste products). In a constant environment, both systems tend towards a time-independent state; and during transience the concentrations of some components may, under suitable conditions, show such phenomena as overshoot and false start. There the similarity between the two systems ends. In a constant environment the amounts of material entering and leaving the open system are equal, and the system does not increase in mass or volume. On the other hand, a bacterial biophase in a suitable constant environment takes in more material than it excretes: the balance is retained within the system which thereby continually increases in mass and volume. Furthermore, a progressively diminishing nutrient concentration will cause an open system to lose mass, or ungrow: whereas a bacterial biophase not only continues to grow under those conditions, but often does so at a rate which hardly diminishes until the medium is almost entirely depleted of nutrients.

\section{EXPANDING SYSTEMS}

When a bacterial biophase grows, it increases its mass at the expense of the medium. But it also increases its volume at the same rate, so that in a medium of constant composition the mean concentration of material in the biophase remains constant. It is clear that if any system is to be a satisfactory kinetic model of the bacterial biophase it must be a delimited entity possessing a capacity for 'endogenous' expansion, whereby it increases its volume and mass at equal rates of its own accord. Furthermore, the growth must be 'balanced' in the sense that the concentration of each component tends to assume a constant value, and does not progressively rise or fall.

Before considering the circumstances under which a system may acquire the capacity for unlimited endogenous or active expansion it may be as well to consider briefly the effect of exogenous or passive expansion on the classical open system.

\section{The effect of expanding an open system}

Reverting to the simple linear system shown in Ex. 1, let it be assumed that the system is in a steady state; that both medium and system have identical solvents; and that the membrane bounding the system is elastic, can be

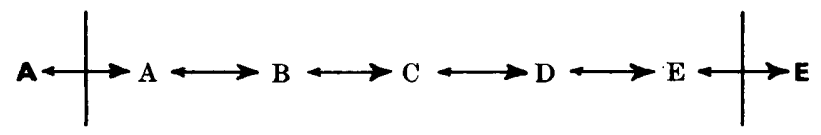


almost instantaneously permeated by the solvent, more slowly permeated by $A$ and $\mathbf{E}$, and is quite impermeable to $B, C$ and $D$.

If the membrane of the system is now stretched so that its volume is suddenly doubled the extra space will be immediately filled by solvent, and the concentrations of all components inside the system will be diluted to half their previous levels. The ratio of flux potentials for components inside the system will remain the same for the instant after expansion; but each flux potential will have half its previous value relative to those of source and sink. The system will in fact be in a condition similar to that described earlier as following a sudden doubling of the concentration of $\mathbf{A}$. As the steady state tends to be re-established the concentration of $A$ will begin to rise, then that of $\mathrm{B}, \mathrm{C}$ and $\mathrm{D}$, and during transition the gradient of flux potentials within the system will descend more steeply than it did in the steady state (Fig. 1).

It is easy to picture what will happen if the volume of the system is increased, not in a sudden extreme step, but gradually and continuously. With an infinitesimally slow expansion the gradient of flux potentials will never deviate significantly from its steady-state value. As the rate of expansion is speeded-up, so the downward gradient of flux potentials will become steeper and steeper, because the concentrations of components inside the system are departing ever further from their steady-state levels. Continuous expansion would lead to a state of affairs in which the amount of each component present at any instant would equal the amount which would have been correct for the steady state of the system when it had some lesser volume. The time elapsed since the system had that lesser volume, or 'delay time', will be progressively larger from one component to the next in order of their kinetic distance from the source.

\section{Endogenous expansion of an open system}

It was shown earlier that, if a compound open system with a branch sequence terminating internally is maintained in a constant environment, the main sequence tends towards a steady state; and if $\mathbf{X}$ and $\mathrm{Z}$ are sufficiently soluble, the branch sequence tends towards false equilibrium. On the other hand, if $\mathbf{Z}$ is insufficiently soluble, $\mathbf{Z}$ becomes an internal sink for the branch sequence which then tends towards its own steady state, with a flux from the internal source $\mathrm{D}$. Of the two cases only the first is truly time-independent: the second is impermanent, because ultimately the fixed volume of the system would be filled by:accumulated insoluble $\mathbf{Z}$.

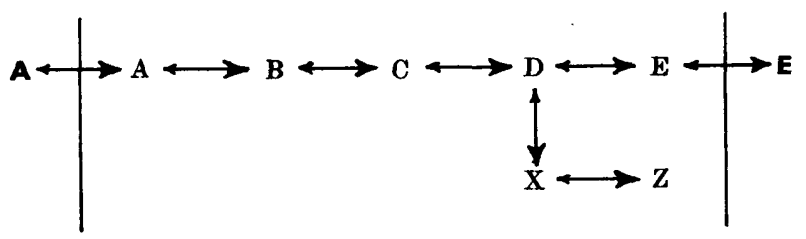

Let it now be assumed that the concentration of $\mathrm{Z}$ cannot rise to a level at which it would be in equilibrium with $X$; and that the volume of the system is proportional to, and controlled by, the total amount of $\mathbf{Z}$. How that 
control is exercised need not be considered at present; but suppose for the sake of representation that $\mathbf{Z}$ forms the boundary membrane which can increase in area by intussusception, and that the shape of the system is such that its surface-area-to-volume ratio does not change during expansion. The zig-zag lines bounding the system in Ex. 10 are used to show that it no longer has a constant volume.

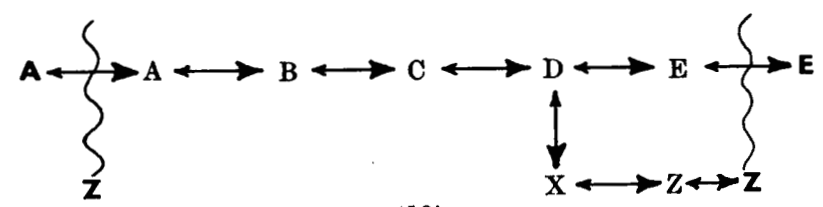

(10)

For a constant concentration of A the flux per unit volume or 'flux rate' through the main sequence will be constant; the concentration of $D$ will be constant, and therefore the rate of formation of $\mathbf{Z}$ per unit volume will be constant. But every increase in the total amount of $\mathbf{Z}$ produces a corresponding increase in the volume of the system. Every increase in volume will in turn lead to an influx of extra $A$ from the medium. The amount of each component in the system will therefore be proportional to the volume; the total rate at which $\mathbf{Z}$ is formed will be proportional to the amount of the components; and the volume of the system will be increasing at the rate at which $Z$ is formed. So at any moment the system will be increasing in mass and volume, or growing, at a rate proportional to its existing mass and volume. The rate of that balanced growth will also depend on the concentration of $\mathbf{A}$; if its concentration be increased the flux rate through the main sequence will increase, the concentration of $\mathbf{D}$ will rise and $\mathbf{Z}$ will be formed more quickly.

\section{The exponential state}

A system such as that considered in the preceding section, which is growing at a rate proportional to its size at any instant, may be described as growing logarithmically because the logarithm of its size plotted against time gives a straight line. The slope of that line is proportional to the 'specific growth rate' of the system.

Continuous expansion of a system has the effect of permanently depressing the concentrations of its components below their constant-volume steadystate level; and the more kinetically remote a component from the source, the greater the disparity in its concentration. During logarithmic growth of a suitably shaped system in a medium of constant composition the components will maintain constant concentrations differing both absolutely and in their mutual ratios from those which would obtain for a constant-volume system in a steady state in the same medium. A system growing logarithmically in a constant environment will tend towards a time-independent condition of balanced growth which may be described as an 'exponential state'. As the ratio of growth rate to flux rate in an expanding system approaches zero, the exponential state approaches the steady state. The steady state can therefore 


\section{New kinetic model of growing bacteria}

be regarded as the limiting case of the exponential state when growth rate tends towards zero, just as equilibrium can be regarded as the limiting case of the steady state when flux tends towards zero.

\section{Autocatalysis in expanding systems}

An expanding system can grow logarithmically and attain the exponential state without benefit of autocatalysis; but since that phenomenon occurs in all living systems so far examined it is interesting to consider the effect of introducing it into an expanding system. A simple example results from assuming that $X$, in the side branch of Ex. 10, is changing to both $Y$ and $Z$; and that $\mathbf{Y}$, or one of its derivatives, acts as $b c$ and catalyses the reaction $\mathbf{B} \longleftrightarrow \mathbf{C}$.

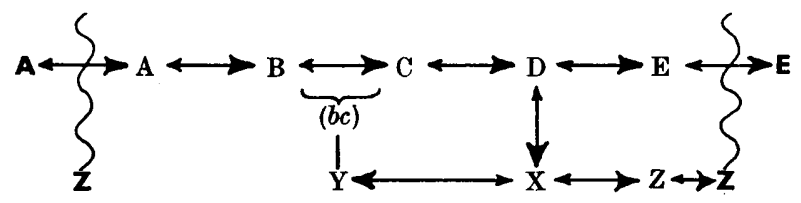

(11)

In a constant environment, $\mathrm{Y}$ tends towards a constant concentration, as do the other components of the system: its concentration cannot increase indefinitely, because each doubling in the amount of $\mathrm{Y}$ involves a doubling of the amount of $Z$, and therefore of the volume of the system. In the simplest case, in fact, $\mathrm{Y}$ is unnecessary and $\mathrm{Z}$ could also be the catalyst $b c$.

Let it be assumed that the uncatalysed reaction $B \leftrightarrow C$ proceeds at a negligible rate, and that the catalyst $\mathrm{Y}$ (or $b c$ ) is initially present in such excess that the rate of decomposition of $B$ is effectively of the first order; the specific growth rate of the system will then depend on the concentration of $\mathbf{A}$. Now let the concentration of $\mathbf{A}$ in the medium be progressively raised, so that the specific growth rate of the system increases. With increasing growth rate the concentrations of all components in the system will rise, but the downward gradient of flux potentials will become increasingly steep. The ratio of the concentration of $Y$, the catalyst, to the concentration of $B$, its substrate, will therefore fall, because $\mathrm{Y}$ is kinetically more remote than $\mathrm{B}$ from the source. When the concentration of $\mathbf{A}$ is sufficiently high, $B$ will be formed more rapidly than the relatively lower concentration of $Y$ can handle it. The catalyst, initially present in excess, will then approach the state of being continuously saturated with its substrate; the reaction $B \longleftrightarrow C$ will approach zero order, and the flux rate through the system will be almost unaffected by further increases in the concentration of $\mathbf{A}$.

The inclusion of an autocatalytic stage in an expanding system therefore permits the flux rate (and thus the growth rate) to tend towards a limiting maximal value as the concentration of source material is increased. It was shown earlier that similar independence does not follow the inclusion of autocatalysis in constant-volume open systems: that difference between the potentialities of the two types of system is of considerable significance. 
The relationship between nutrient concentration and growth rate

It is now possible to forecast the general form of the relationship between nutrient concentration, [A], waste-product concentration, [E], and specific growth rate during the exponential state, $\bar{\gamma}$, for an autocatalysed expanding system of the type shown in Ex. 11. If the absolute concentration of $\mathbf{A}$ be $a$ and its concentration when in equilibrium with the given value of [E] be $a_{\mathrm{e}}$, then $a-a_{\mathrm{e}}=a_{\mathrm{x}}$, the 'effective concentration' of A. When $a_{\mathrm{x}}$ is negative $\bar{\gamma}$ will be negative, and the system will 'ungrow'; when $a_{\mathrm{x}}$ is zero, $\bar{\gamma}$ will be zero; and when $a_{x}$ is positive $\bar{\gamma}$ will be positive, and the system will grow. The value of $\bar{\gamma}$ will have a first-order relationship with small positive values of $a_{x}$; but will approach a zero-order relationship with successively larger values of $a_{\mathbf{x}}$, tending asymptotically towards a limiting maximum value, $\bar{\gamma}_{\mathbb{M}}$.

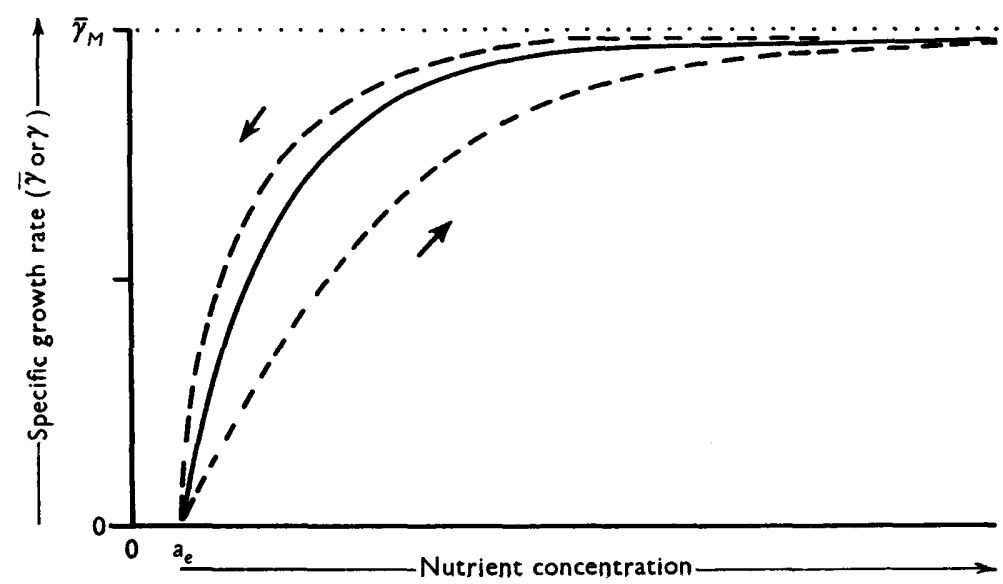

Fig. 2. Growth-rate hysteresis : curves showing the general form of the predicted relationships between nutrient concentration and specific growth rate in an autocatalysed expanding system: —_, curve for the limiting growth rate, $\bar{\gamma} ;---$, curves of ascent and descent for the transient growth rate, $\gamma ; \ldots . .$, maximum growth rate, $\bar{\gamma}_{M} ; a_{\mathrm{e}}=$ concentration of source material in equilibrium with sink material.

When the system is surrounded by a medium in which nutrient concentration is varying, the values of $a_{x}$ and $\bar{\gamma}$ will therefore be related by a curve of the general form shown by the solid line in Fig. 2 only if the rate of change of $a_{x}$ is so slow that the system never departs significantly from the exponential state. On the other hand, if $a_{x}$ is changing relatively rapidly, the concentrations of components within the system will not have time to reach their exponential state levels, and the 'transient specific growth rate' of the system, $\gamma$, will be less than $\bar{\gamma}$ when $a_{x}$ is rising and greater than $\bar{\gamma}$ when $a_{x}$ is falling: I call that phenomenon 'growth-rate hysteresis' (Perret, 1956). The extent and sign of the difference between $\gamma$ and $\bar{\gamma}$ at any instant will therefore depend on the manner in which $a_{x}$ has been changing; and the single curve will be replaced by families of pairs of curves corresponding to ascending and descending values of $a_{x}$ (Fig. 2). 


\section{The effect of sudden alterations in the medium}

Consider the case of two media containing the same nutrients at different concentrations, and let the limiting specific growth rate of an autocatalysed expanding system during balanced growth in the 'strong' medium be $\bar{\gamma}$ ', and in the 'weak' medium $\bar{\gamma}$ ". It is easy to forecast the probable result of transferring the system in an effectively instantaneous manner from one medium to the other, under circumstances which avoid any additional traumatic events.

If the concentrations of nutrient in both the strong and the weak media, although very different, are nevertheless so high that both $\bar{\gamma}^{\prime}$ and $\bar{\gamma}^{\prime \prime}$ are virtually equal and undetectably different from the maximum growth rate, $\bar{\gamma}_{\mathbf{M}}$, then neither the weak-to-strong nor the strong-to-weak transfers would produce any noticeable change in the rate of expansion of the system, and growth would continue unchecked. But if the concentrations of nutrient in the two media are such that $\bar{\gamma}^{\prime}$ is significantly greater than $\bar{\gamma}^{\prime \prime}$ the effect of the transfer would depend on its direction. A change from strong to weak medium would be followed by a period of deceleration of growth rate, while the concentrations of the components of the system declined towards their new exponential state levels. The reverse change, from weak to strong, would be followed by a period of accelerating growth rate as the concentrations of components increased. However, it appears that decreases in concentration will be assisted, and increases hindered, by the diluting effect of continued expansion: so it might be expected that periods of acceleration would be relatively extended; and periods of deceleration relatively curtailed, perhaps to an extent where the adjustment of growth rate appeared to be virtually instantaneous.

A second type of transfer is possible if the two media contain, not the same nutrients at different concentrations, but alternative nutrients, as represented by $\mathbf{A}$ and $J$ in Ex. 12.

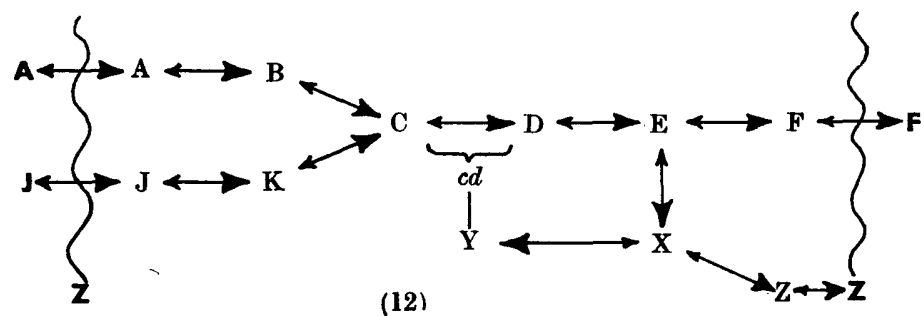

In this case, if the medium containing A permits a higher growth rate than the medium containing $J$, the media may be described respectively as rich and poor. But both the rich-to-poor and poor-to-rich transfers will be followed by periods in which the concentrations of new intermediate metabolites ( $\mathrm{A}$ and $\mathrm{B}$, or $\mathbf{J}$ and $\mathrm{K}$ ) are rising to their exponential state levels. It might therefore be expected that the transfer in either direction would commonly be followed by a period of accelerating specific growth rate, necessarily 
preceded by a short period of decelerating growth rate after the rich-to-poor transfer.

\section{Growth of an autocatalysed expanding system in a finite amount of nutrient}

It has so far been assumed that the consumption of nutrient by the expand- . ing system has no effect on the composition of the medium. But most studies on the growth of bacterial biophases have been made in batch culture where the ultimate mass of the biophase is limited by the amount of nutrient present. It therefore seems appropriate to end this part of the paper by drawing together various threads from the preceding arguments and considering the probable behaviour of an autocatalysed expanding system under similar conditions. The system to be used as an example is represented in Ex. 13.

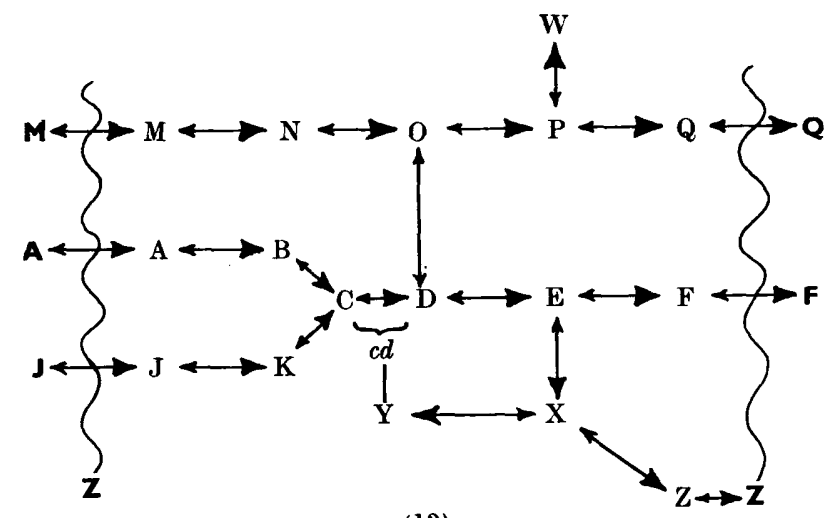

(13)

It will be assumed that $\mathbf{A}$ and $\boldsymbol{J}$ are two alternative 'major nutrients', and that the sequences $\mathbf{A}, \mathbf{A}, \mathbf{B}, \mathbf{C}-\mathbf{F}$ or J, J, K, C-F are alternative 'major pathways' almost entirely in control of the growth of the system. $M$ is a 'minor nutrient' metabolized via a 'minor pathway' to $\mathbf{Q}$; the components $\mathbf{M}, \mathbf{N}, \mathbf{O}, \mathbf{P}, \mathbf{W}$ and $\mathrm{Q}$ contribute negligibly to the total mass of the system; and the coupling $\mathrm{O} \longleftrightarrow \mathrm{D}$ is so loose that the flux rates through the major and minor pathways are almost independent of each other. The expanding system is to be transferred from a medium containing either $\mathbf{A}$ or $\mathbf{J}$ to one containing A at a concentration such that $\bar{\gamma}$ will be indetectably smaller than $\bar{\gamma}_{\mathbf{M}}$.

Immediately after transfer to the new medium the system will enter a transient 'phase of adjustment' of a type depending on the nature of the previous medium. That phase may be undetectable and short; or it may be marked and prolonged, in which case it is most likely to consist mainly of a period during which specific growth rate is accelerating from a low to a higher level.

The phase of adjustment, if it occurs, will ultimately merge into a phase of logarithmic growth which may or may not be, in its early stages, very nearly a phase of balanced growth, with the system almost in an exponential state. But as the logarithmic phase progresses the concentrations of nutrients will fall, and of waste products rise, at logarithmically increasing rates. The con- 
centrations of metabolites on the minor pathway, and the flux through them, may therefore vary widely long before the falling concentration of $\mathbf{A}, a_{\mathbf{x}}$, has led to a detectable diminution of the magnitude of $\bar{\gamma}$. Growth, although still logarithmic, will then be far from balanced, and the system as a whole will be far from the exponential state.

The value of $a_{x}$ will eventually fall so low that specific growth rate becomes detectably less than $\bar{\gamma}_{\mathbf{M}}$ and diminishes progressively towards zero. During that phase of retardation the system will have a falling transient specific growth rate, $\gamma$, always slightly above the limiting specific growth rate, $\bar{\gamma}$, for the corresponding ambient concentration of A. Finally, when $a_{x}$ has fallen to $a_{0}$, growth will cease, and the system will enter a stationary phase in which its final mass and volume will depend on its size at the time of transfer and on the initial concentrations of $\mathbf{A}$ and $\mathbf{F}$ in the medium.

During the stationary phase metabolism will have effectively ceased in the major pathway. However, if the medium still contains $M$, metabolism may continue vigorously in the minor pathway, which will then resemble a linear constant-volume open system. If the system is subjected, before it has reached the stationary phase, to some treatment which blocks the formation of $\mathrm{Z}$, growth will cease. But metabolism will continue in both the major and minor pathways, and the system will behave as a compound reticular constantvolume open system.

\section{THE EXPANDING SYSTEM AS A MODEL BACTERIAL BIOPHASE}

If the arguments developed in the preceding parts of this paper are correct, then a relatively simple expanding system cannot only exhibit balanced or unbalanced logarithmic growth but can also behave in a manner remarkably reminiscent of the lag, acceleration, logarithmic, retardation and stationary phases of a bacterial batch culture. Furthermore, those similarities of behaviour do not need to be specially contrived, but are implicit in the kinetic structure of the system; a structure basically very similar to the known kinetic structure of living cells. It therefore seems reasonable to propose that the type of system shown in Ex. 13 is in essence, and within its obvious limitations, a valid elementary kinetic model of a bacterial biophase. Acceptance of that proposal justifies re-examination of the relevant concepts of theoretical microbiology in the light of the properties of expanding systems. A few important examples of the results of such re-examination are considered below.

\section{The concept of the 'master reaction'}

The concept of the 'master reaction' is usually attributed to Blackman (1905) who stated, without proof, that "when a process is conditioned as to its rapidity by a number of separate factors, the rate of the process is limited by the pace of the 'slowest' factor". That statement was attacked by Burton (1936) in a paper which discussed "the principle that the rate of a chain of processes is determined dominantly by the speed of the slowest process in the chain" and concluded that "in the steady state of reaction chains the principle 
of the master reaction has no application" and therefore, by implication, that Blackman was wrong.

Burton's dismissal of Blackman's axiom seems to have been commonly accepted as the last word on the matter, in spite of the fact that it was based on the attributes of sequences of irreversible uncatalysed first-order transformations, whereas the reactions in biological systems are generally reversible, catalysed, and of all feasible orders from virtually zero upwards. But a more important discrepancy seems also to have escaped notice. Blackman stated that rate was 'limited', not 'dominantly determined', by the 'slowest' reaction; the text of his paper shows clearly that he was fully aware of the important distinction between limitation and determination, and that the word 'slowest' was used in a special but acceptable sense. The properties of a convoy of vehicles provide an analogy. The submaximal speeds of such a convoy may be determined by the interplay of many factors; but if one vehicle has a lower maximum speed than the others it may be described as the 'slowest'. All vehicles in the convoy travel at the same speed, but the 'slowest' limits the possible speed of the convoy.

It was shown earlier that the 'rate-limiting' type of master reaction can result from the introduction of extraneous catalysis into a constant-volume open system, or of autocatalysis into an expanding system. Blackman's statement was therefore correct, but its misinterpretation and apparent disproof by Burton have had an unfortunate effect on the development of theoretical biology.

\section{The logarithmic phase of growth in batch culture}

The growth of a bacterial biophase is usually measured in terms of its increase in dry weight, or of some property bearing a known relationship to dry weight. The logarithmic phase of growth may therefore be described as that period during which the sum of the masses of all cellular components not substantially vaporized at about $100-110^{\circ}$ is increasing at a rate which, within the limits of experimental error, is more or less proportional to its own magnitude.

Monod (1949) expressed the generally accepted interpretation of the nature and implications of the logarithmic phase in the words "it is reasonable to consider that a steady state is established, where the relative concentrations of all the metabolites and all the enzymes are constant. It is, in fact, the only phase of the growth cycle when the properties of the cells may be considered constant and can be described by a numeric value, the exponential growth rate, corresponding to the over-all velocity of the steady state system." If the bacterial biophase is regarded as a delicately integrated autosynthetic system in which flux rate or growth rate depends on the concentration of every compound because rate-limiting master reactions are impossible, some such interpretation of the logarithmic phase seems unavoidable. Yet it is difficult under those circumstances to imagine how all internal concentrations remain so constant in the face of widespread alterations in the composition of the medium; and even more difficult to explain away the extensive evidence 


\section{New kinetic model of growing bacteria}

that the properties of the cells are not constant during the logarithmic phase, and that the concentrations of enzymes and metabolites often vary considerably. Finally, the term 'steady state' as applied to an open chemical system normally means that the rates at which material is entering and leaving each unit volume are equal, whereas 'growth' implies that the inflow per unit volume exceeds the outflow.

All those difficulties disappear if the logarithmic phase is reinterpreted in terms of the properties of a reticular expanding system which contains at least one autocatalytic stage. The existence of a rate-limiting master reaction then becomes possible, and a virtually constant specific growth rate is quite compatible with substantial changes in the concentration of some enzymes and metabolites. The proportions of individual components of the system can vary (provided that the sum of their masses per unit volume remains constant within the limits of experimental error) because the time-independent exponential state is attained by an expanding system only when the environment is constant.

\section{The kinetics of bacterial metabolism}

The misconception that constancy of specific growth rate during the logarithmic phase in batch culture implies constant composition of the bacterial biophase has led to the performance of numerous experiments of doubtful value and to some debatable conclusions. For instance, many studies of the kinetics of enzyme induction (e.g. Pollock, 1952, 1953) depend for their interpretation on the assumption that the biophase was in a virtually steady state during logarithmic growth. That argument was always open to the objection that induction must disturb the steady state in at least the part of the system under investigation, and a disturbed steady state is not necessarily more tractable than a disturbed unsteady state. To that objection may be added, in the best forensic manner, the further objection that the state was probably unsteady before it was disturbed, if constancy of growth rate was the only criterion of steadiness (Perret, 1956). One is faced with the paradoxical situation that detailed kinetic interpretations of the results of such enzyme induction experiments are justified only if the experiments cannot be performed. As Blackman (1905) wrote "the way of those who set out to evaluate exactly the effects of changes in a single factor upon a multi-conditioned metabolic process is hard".

Two conditions must be satisfied if experiments depending for their validity on the constancy of composition of the biophase are to be carried out on batch cultures: specific growth rate must be constant and the environment must be constant. In order to justify the assumption that the biophase has a virtually time-independent composition it is customary to use a minute, fully adapted inoculum; and to end the experiment while the vast majority of nutrient remains unconsumed. But in batch culture relatively large changes in the concentration of waste products occur at the time of inoculation, and during the early stages of growth. It is therefore safer in principle to take the further precaution of adding to the uninoculated medium a proportion of waste products such that their concentration is also virtually constant during 
the experiment. Inability to carry out the necessary measurements on a very dilute suspension of organisms should be met by performing the experiments on homocontinuous cultures, and not by ignoring the precautions which justify the use of batch cultures.

\section{Nutrient concentration, specific growth rate and yield}

A simple equation describing the empirical relationship between the ambient concentration of a limiting nutrient and the specific growth rate of a bacterial biophase during logarithmic growth was proposed by Monod (1942) for various bacteria growing on simple carbohydrates, and has since been shown to have wider applications (Novick, 1955). If $a_{\mathrm{k}}$ is the concentration of limiting nutrient which permits a specific growth rate of half the maximum value, and the other symbols have the meanings allotted earlier, the equation is

$$
\bar{\gamma}=\frac{\bar{\gamma}_{\mathrm{M}} a}{a_{\mathrm{k}}+a}
$$

which generates curves similar in shape to that shown as a solid line in Fig. 2, but passing through the origin.

Monod clearly intended his equation to apply only to limiting growth rate, $\bar{\gamma}$, and not to transient growth rate, $\gamma$. Unfortunately the origin of the equation has been overlooked by some workers in recent years and the expression has been treated as though it were a relationship between $\gamma$ and $a$, which it cannot be, because of the existence of growth-rate hysteresis. That confusion has led to some minor errors in the mathematical theory of homocontinuous culture which, in its present form, is true only for established time-independent states and cannot be used for the detailed prediction of events during transience or transition. For instance, the proof by Spicer (1955) that a homocontinuous culture system cannot overshoot the steady state is not strictly valid (Perret, 1956). In fact, the existence of growth rate hysteresis seems to allow the possibility of persistent instability appearing as intermittent growth, accompanied perhaps by some degree of synchronized cell division, at very low dilution rates in homocontinuous culture (Perret, 1956). In order to avoid future misunderstandings it seems advisable to use the term 'limiting growth rate' and a special symbol, such as $\bar{\gamma}$, which emphasize the restricted purpose for which Monod proposed the equation.

To suggest the replacement of $a$ in Eqn (1) by $a_{e}$, the effective concentration of nutrient, may seem absurdly pedantic because of the very large changes in free energy normally associated with the breakdown of bacterial nutrients into waste products. But future investigations may disclose some forms of metabolism involving relatively small free energy changes and having correspondingly low values for the overall equilibrium constant. The effect of the concentration of waste products might then show up, particularly during attempts to grow large populations of bacteria at very low growth rates in homocontinuous culture, as a tendency for the value of $\bar{\gamma}$ to become zero in the presence of a detectable ambient concentration of nutrient. In such cases, if they ever occur, it would also be necessary to use $a_{\mathrm{e}}$ in Monod's equation for the relationship between nutrient concentration and yield (Monod, 1942). 


\section{EXPANDING SYSTEMS AND THE ORIGIN OF LIFE}

\section{The nature of simple organisms}

That the concepts 'life' and 'living' cannot be strictly defined has been repeatedly emphasized (e.g. Lotka, 1924; Pirie, 1937). However, it is possible to list some of the properties shared by all those types of simple system yet investigated which are able to thrive in a non-living environment and are unanimously accepted as being alive. Whether any one of those attributes is so crucial that a system lacking it should be classified as non-living is largely a matter of personal choice.

Primary attributes. The organisms are delimited entities, set apart from their chemical environment, or medium, by a membrane or interface. Their chemical composition differs quantitatively from that of the medium, is often relatively unaffected by changes in the medium, and is made up of many different substances of which some are in solution.

Secondary attributes. The organisms have three attributes which jointly allow them to participate in evolutionary changes.

(i) 'Balanced growth': the organisms are able to increase their mass and volume at the expense of the medium in such a manner that their average composition often remains virtually constant for substantial periods of time.

(ii) 'Division': the organisms are able to maintain a constant average size in spite of growth by dividing into, or giving rise to, daughter organisms sharing the characteristics of the parent cell.

(iii) 'Persistent variation': the chemical composition of individual organisms is able to undergo seemingly random changes which then often persist in the organism and its progeny for many generations.

Tertiary attributes. The organisms partially consist of numerous large and complex molecules of types not known to be formed at a significant rate by non-living systems. Some of these molecules are so linked together that they occupy more-or-less fixed relative positions in the cell and form an apparently important three-dimensional pattern. Many reactions occurring inside the cell are selectively catalysed by particular macromolecules whose structure is responsible for their specific catalytic properties. The important structural details of the different species of macromolecules and the general structure and composition of the cell appear to be controlled by, or associated with, the detailed structure of specialized macromolecules which are themselves the products of cellular metabolism. The whole cell seems therefore to be a delicately integrated autosynthetic system in which the formation of each molecular species is directly or indirectly mediated by all the other molecular species, the control system being a stabilized closed loop.

\section{The essential attributes of an eobiont}

All the primary attributes are possessed by many chemical systems unanimously accepted as non-living; for instance, by a droplet of oil suspended in an aqueous solution of organic and inorganic compounds. But the only secondary attribute commonly conceded to such systems is the capacity for 
passive division; so that balanced growth, persistent variation and the tertiary attributes are regarded as peculiar to living organisms. Furthermore, experimental evidence suggests that the tertiary attributes of organisms are actually involved in their manifestation of the secondary attributes. As a result it seems to have been generally assumed that some of the ubiquitous tertiary attributes must be 'essential prerequisites' for a system to grow, divide and vary in the manner of living organisms. That assumption has been implicit in most theories about the sequence of events in 'biopoesis' (Pirie, 1953), having led to considerable argument about which aspect of the tertiary attributes is the key to the origin of life, and how it might have arisen spontaneously in a non-living environment.

However, an alternative and more economical approach to the problem follows the assumption that the tertiary attributes are not necessary for growth, division and variation, but are refinements enabling those processes to occur with efficiency and precision; the consequences of evolution, not the means by which it occurred. A system possessing the primary and secondary attributes, and placed in a suitable environment, could seemingly develop all the tertiary attributes in the course of evolution. If that possibility be conceded, the problem then is to suggest one or more reasonable ways in which a non-living chemical system lacking the tertiary attributes might spontaneously acquire the capacity for the right kind of growth, division and variation, and so become qualified for consideration as an 'eobiont' (Pirie, 1953).

The expanding system as a possible eobiont

The case for probiotic evolution, by which the existing types of microorganism might have evolved from simpler systems, was developed by Oparin $(1938,1957)$, who realized the importance of thinking in terms of delimited droplets, and not in terms of reactions in homogeneous solution or of imaginary 'self-reproducing' molecules. But Oparin's suggestions suffered from one or two minor deficiencies; his droplets were coacervates, which generally seem to require proteins, nucleoproteins or polysaccharides for their formation: and he made no detailed proposals about the manner in which they might have acquired the important property of balanced growth. The expanding system concept seems to round off Oparin's ideas by suggesting a way in which balanced growth could have occurred in systems so much simpler than his coacervates that their spontaneous formation would presumably be more probable.

Since an organism can contain only those chemical elements present in its medium, it follows that all reactions occurring inside an organism can also spontaneously occur outside, although they may actually do so at quasiinfinitesimal rates. Differences between the composition of a growing organism and its medium are therefore maintained by differences between internal and external rates of reaction. Such rate differences could be due to catalysis, to the inequalities of concentration associated with immiscible phases or, of course, to both. Catalysis appears to be the major factor in known organisms, and it could also have been the primary factor in the formation of eobionts; 
but that supposition has certain disadvantages. For instance, it calls for reasons to account for the non-occurrence of similar catalysis with equal frequency in the surrounding medium; it raises the problem of delimitation of the eobiont; and seems generally to involve a degree of complication comparable with that of known unicellular organisms, because the formation of every component having a higher internal than external concentration would have to be directly or indirectly catalysed. It therefore appears more inviting to assume that the differential reaction rates of eobionts were probably associated with immiscible phases, and that catalysis was acquired by easy stages in the course of evolution.

For the sake of diagrammatic simplicity the volume of an expanding system was represented earlier as being restricted by a membrane; the state of affairs which would exist in the familiar 'chemical garden' if the substances forming insoluble silicates could be generated inside the 'growths' by the exergonic reaction of soluble compounds diffusing in from the medium. But the same arguments apply also to systems in which the volume-controlling component forms a phase immiscible with the medium: such systems could then be delimited by either a simple phase boundary or a membrane. In the case of an immiscible phase delimited by a phase boundary it is easy to suggest a simple type of expanding system which it ought to be possible to study in the laboratory. Of the innumerable pairs of partially miscible substances able to form 'conjugate solutions' the familiar phenol-water system is here used as an example, without in any sense suggesting that it was actually concerned in biopoesis.

Consider a large volume of water containing numerous organic and inorganic compounds in dilute solution; and assume that two of the compounds could take part in a markedly exergonic reaction leading to the formation of phenol. At temperatures below the consolute temperature the concentration of phenol could rise until its solubility was exceeded, whereupon the system would separate into two phases: droplets of water-saturated phenol in a phenolsaturated aqueous medium. Following droplet formation all the compounds in the medium would become distributed between the two phases in accordance with their differential solubilities. The quantitative chemical composition of the phenol droplets would then differ considerably from that of the aqueous medium in respect of many components. Where internal concentrations became much higher than external concentrations, reactions occurring extremely slowly in the medium might occur relatively rapidly inside the droplets, thereby accentuating the differences in composition. Other automatic consequences of droplet formation would be such general colloidal phenomena as the orientated concentration of surface active molecules at the interface, the formation of a hydration layer and the adsorption of ions: changes which would affect the stability of the droplets and selectively modify rates of inward and outward diffusion.

In order to endow the droplets with the capacity for growth from within it is now merely necessary to make the reasonable assumption that the partition coefficient of the phenol precursors could be such that their concentration inside the droplets exceeded the level which would be at equilibrium 
with the internal concentration of phenol. The reaction of the precursors inside the droplets would then lead to the formation of more phenol, and the droplets would grow. Further precursor molecules would then diffuse in to replace those which had reacted, and growth would continue for as long as the supply of precursors in the medium was maintained. But every increase in volume and mass of the system would be followed by the inward diffusion of all the other components in order to maintain their distribution ratio. Growth would therefore be almost balanced; but not quite so, because the diminishing surface-area-to-volume ratio of the droplet would adversely affect its growth rate and lead to progressive alterations in its composition.

\section{The evolution of droplet expanding systems}

Before droplet-expanding systems could evolve by natural selection they would need, in addition to growth, the further attributes of division and persistent variation. Since passive division would be adequate for the initial stages of evolution, its occurrence presents no problem. Each droplet would be held together by surface tension, but its physical instability would increase with size; chance hazards, such as turbulence in the medium, would therefore be likely to disrupt the larger droplets; and disruption would be assisted if the gradual changes in composition during growth happened to lead to a decrease in surface tension. The virtual homogeneity of the droplets and the absence of specialized structures needing to be distributed between the progeny would make it highly probable that daughter droplets would share their parent's capacity for growth.

It appears that neither the formation of the expanding droplets nor their growth and division involve any events of a degree of improbability high in the context of biopoesis. It now remains to suggest a reasonably probable way in which persistent variation might occur. Any change in the chemical composition of a droplet would necessarily affect its surface tension and solvent properties, and would thereby alter, no matter how slightly, its physical stability and its efficiency of growth and division. But the more specialized the composition of the droplets, the greater the chance that any random change would be harmful; and the frequent occurrence of harmful persistent changes might lead to the destruction of the droplet population, rather than to its evolution. Suitable random variations would therefore need to be not only persistent but also rare, in the sense of affecting only a small proportion of the droplets in any one 'generation'. It appears that randomness and persistence, together with the necessary nice balance between the extremes of probability and improbability, might be provided by the simplest form of autocatalysis, in which the possession of one molecule of a given species by a droplet would increase the chance of its acquiring further molecules of the same species.

The theory of chemical reactions does in fact predict that, in a constant environment, each droplet would occasionally form within itself (or acquire from the medium by chance encounter) single molecules of various rare species. The qualitative composition of each droplet would therefore vary slightly from one moment to the next; and different droplets would have slightly 


\section{New kinetic model of growing bacteria}

different compositions at the same instant. Such differences would, however, be transitory as well as random, and would not lead to progressive alterations in the composition of the droplet population or to the formation of persistently variant 'clones'. But if one of the possible types of rare molecule were endowed with the necessary autocatalytic property it could 'multiply' until it reached an exponential-state concentration, and then form a persistent component of one droplet and its progeny. In cases where the change was beneficial the variant clone could outgrow the unchanged droplets and primitive evolution would occur.

It is obvious that such an eobiont would gain great advantages from the acquisition of a selectively permeable boundary membrane, numerous autocatalysts, an internal structure favouring a spatially ordered sequence of metabolic reactions, a hereditary 'code-script' (Schrödinger, 1944), mechanisms favouring regular fragmentation and other devices for maintaining the composition of the system in a highly efficient state. But the possible properties of droplet-expanding systems seem to lend weight to the suggestion that those attributes could be the result of millions of years of evolution, starting at an even more primitive level than is implied by Oparin's coacervates (Pirie, 1953; Lindegren, 1955).

\section{DISCUSSION}

The phenomenon of sustained balanced growth of a biophase has been interpreted as the expression of a complex autocatalytic integration of chemical reactions, of a type sometimes called 'autosynthetic', in which the rate of formation of every component of the system is directly or indirectly dependent on the amount of every other component. It has been shown that, in a constant environment, such autosynthetic systems will ultimately settle down into so-called 'steady states' in which the amounts of all components increase at a common exponential rate (Hinshelwood, 1952; 1953 $a, b$ ).

Expanding systems are certainly autosynthetic in the general sense that they autonomously mediate the formation of more of themselves; but, since they are not autosynthetic in the specific sense used by Hinshelwood, they deserve a distinctive name. General chemical interdependence between all the components of an expanding system is quite unnecessary; and even 'passive' components taking no part in the reaction sequences share the common exponential increase. The crucial assumption in the expanding system concept is merely that the volume of an open system can be proportional to the amount of one or more reaction products forming an internal sink. It can easily be shown, by either mathematics or analogy, that such a simple system will also settle down into a temporally-invariant state similar to that resulting from the complexities of autosynthetic integration. But the expanding system approach reveals that balanced growth can occur in the absence of any autocatalytic stage, and therefore, by implication, that enzymes are not essential for the type of growth or self-reproduction possessed by living systems. 
During the steady state the amounts of material entering and leaving a constant-volume system are equal; but the efficiency of conversion of nutrients to cell-substance by a bacterial biophase may be $35 \%$ (Monod, 1949) or more. That means, in effect, that for every two molecules of nutrient leaving unit volume of the biophase in modified form, one or more molecules may be retained internally. Furthermore, a constant-volume system in its steady state cannot have internal sinks, so there must be a flux through every component such that its rates of formation and breakdown are always equal; but that is known to be quite untrue of most components of a growing biophase. It is therefore obvious that, even in a constant environment, neither the growing biophase as a whole, nor each unit volume of it, could possibly be in the kind of steady state typical of a constant-volume system. The interpretation of the kinetics of growing biophases in terms of constant-volume steady states has caused confusion, and even done considerable harm, in some fields of practical and theoretical microbiology. In particular, it has delayed recognition of the fact that growth rate may be limited by a single master reaction.

Both chemical equilibrium and the steady state are conditions in which concentrations do not vary with time; but the two states have other properties which are dissimilar, so they are distinguished by different names. The temporally-invariant condition of autosynthetic and expanding systems is a third state of constant concentrations, but one in which amounts are increasing exponentially; it therefore seems useful to emphasize its distinctive properties by calling it the 'exponential state'. The steady state is, in a sense, a condition of permanent transience towards an unapproachable equilibrium; the exponential state is, in a similar sense, a condition of permanent transition towards an unapproachable steady state. A bacterial biophase will attain a true exponential state only during growth in a constant environment; that is, under the conditions which would exist in an ideal homocontinuous culture. The exponential state could also be nearly attained during logarithmic growth in batch culture, under suitable conditions; but a mass of experimental evidence throughout the literature suggests that such conditions are rarely realized in practice. The inclusion of a single autocatalytic stage in an expanding system permits it to manifest a logarithmic phase of growth under the conditions of batch culture without entering, or even nearly approaching, the exponential state.

The expanding system is put forward primarily as a kinetic model of growing bacteria as they are now, with all their interdependent complexities of cellular and molecular structure: such complexities can be accommodated within the model system, and could clearly be to its advantage. But the system can apparently function without those complexities, and there seems to be no need to endow its hypothetical molecular species with the properties peculiar to proteins, enzymes, nucleoproteins, polysaccharides or the adenosine phosphates; or to allocate to the molecules any fixed spatial relationships; or to assume that their mechanisms of formation involve any elaborately coordinated mutual dependence. The two aspects of the nature of expanding 
systems which have the widest biological significance are that they can grow indefinitely, in a balanced manner, without any autocatalytic process; and that the virtual constancy of their composition in respect of every component is maintained without any complex 'code-script' to direct and coordinate their behaviour.

The type of self-reproduction found in living organisms is sometimes assessed as their crucial peculiar property; and the nature of self-reproduction has been discussed in terms of 'information theory' (e.g. Haldane, 1954; Penrose \& Penrose, 1957). That attitude seems to involve the implied assumption that an organism engaged in self-reproduction has a 'choice' of courses before it and needs to be 'instructed' which to take by some specialized molecules. However, the expanding system concept suggests that control of the composition of cytoplasm could be entirely autonomous, although it appears not to be so in modern organisms. Self-reproduction can be the inevitable consequence of one or more normal chemical reactions which, in endeavouring to approach equilibrium, provide both the material and energy needed for expansion: a phenomenon no more mysterious or peculiar than the accumulation of diffusible solutes from an aqueous medium by a water-filled dialysis bag, or the partition of iodine in the correct ratio between water and chloroform phases. The system behaves as it does because it cannot behave otherwise; and the only 'information' needed for the continuous reproduction of its substance is that controlling all chemical reactions; the information implicit in the nature of energy and matter. The information-theory approach may be valuable when applied to the possible inter-relationships between the structural details of proteins and nucleic acids, or to autosynthetic systems whose self-reproductive powers depend on the specificity of enzymes: but it may actually obstruct our analysis of the general phenomenon of chemical self-reproduction. For instance, the often-quoted proof that a self-reproducing machine can be constructed from about twelve different kinds of components (von Neumann, 1951) is hardly relevant to chemical reproduction, because an expanding system of perhaps only two components, or certainly of three (e.g. water, and two substances able to react spontaneously in an aqueous phase to form water) could reproduce itself indefinitely in a suitable environment.

It was suggested earlier that arguments about the nature and possible origin of life have been bedevilled by the unjustified assumption that ubiquitous necessarily implies essential: the idea that simple is synonymous with primitive has caused similar confusion. If viruses are classed as living, then some of them are undoubtedly the simplest known living organisms. But, far from being primitive, they seem to be the ultimate stage of obligate intracellular parasitism. It is absurd to apply to viruses, genes or nucleic-acid-containing molecules in general the unmerited epithet 'self-duplicating', and then to assume that they could exercise that disputed capacity in a non-living medium, when all the available experimental evidence points clearly to the contrary conclusion. Even if nucleic acids or nucleoproteins are eventually shown to possess autonomous powers of self-duplication in a non-living medium, there 
would still be much to recommend the hypothesis that biopoesis occurred through 'cellular' systems: meanwhile, that hypothesis seems to be the only one scientifically justified.

The spontaneous formation and survival of expanding-system eobionts composed of organic compounds would be virtually impossible in natural environments nowadays, because of competition and predation by established organisms. But there seems to be no reason why expanding systems should not be formed and survive in suitable sterile media. Tests of sterility based on the properties of highly developed micro-organisms could not be expected to detect, or reveal as 'living', the kind of minute, structureless, extremely slow-growing and chemically simple objects which might be the early stages of biopoesis. But, knowing what to expect, the intentional formation of expanding systems and the experimental examination of their growth and variation seems not to involve any formidable practical difficulties.

\section{REFERENCES}

Blackman, F. F. (1905). Optima and limiting factors. Ann. Bot., Lond. 19, 281.

Burton, A. C. (1936). The basis of the principle of the master reaction in biology. J. cell. comp. Physiol. 9, 1.

Burton, A. C. (1939). The properties of the steady state compared to those of equilibrium as shown in characteristic biological behaviour. J. cell. comp. Physiol. 14, 327.

DANCKWERTs, P. V. (1954). Continuous flow of materials through processing units. Industr. Chem. Mfr, 30, 102.

Dean, A. C. R. \& Hinshelwood, C. N. (1955). Reaction patterns of a coliform organism. Progr. Biophys. 5, 1.

Denbigh, K. G. (1944). Velocity and yield in continuous reaction systems. Trans. Faraday Soc. 40, 352.

Denbigh, K. G. (1947). Continuous reaction. II. The kinetics of steady-state polymerisation. Trans. Faraday Soc. 43, 648.

DenBigh, K. G. (1951). The Thermodynamics of the Steady State. London: Methuen.

DENBIGH, K. G. (1952). Entropy creation in open reaction systems. Trans. Faraday Soc. $48,353$.

Denbigh, K. G., Hicks, M. \& Page, F. M. (1948). The kinetics of open reaction systems. Trans. Faraday Soc. 44, 479.

Denbigh, K. G. \& PAge, F.M. (1954). The capacity flow method in chemical kinetics. Disc. Faraday Soc. 17, 145.

Hardane, J. B. S. (1954). The origins of life. New Biol. $16,12$.

Herbert, D., Elsworth, R. \& Teluing, R. C. (1956). The continuous culture of bacteria; a theoretical and experimental study. J. gen. Microbiol. 14, 601.

Hinshelwood, C. N. (1946). The Chemical Kinetics of the Bacterial Cell. Oxford: Clarendon Press.

Hinshelwood, C. N. (1952). On the chemical kinetics of autosynthetic systems. $J$. chem. Soc. p. 745.

Hinswelwoon, C. N. (1953a). Alternative reaction patterns in autosynthetic systems. J. chem. Soc. p. 1304.

Hinshelwood, C. N. (1953b). Autosynthesis. J. chem. Soc. p. 1947.

Lindegren, C. C. (1955). Is the gene a prime mover? Nature, Lond. 176, 1244.

Lotka, A. J. (1924). Elements of Physical Biology. Baltimore: Williams and Williams.

Monod, J. (1942). Recherches sur la croissance des cultures bactériennes. Paris: Hermann et Cie. 
Monod, J. (1949). The growth of bacterial cultures. Annu. Rev. Microbiol. 3, 371. Monod, J. (1950). La technique de culture continué: théorie et applications. Ann. Inst. Pasteur, 79, 390.

Moser, H. (1957). Contributions to the theory of the continuous bacterial growth apparatus. I. Kinetics of growth of homogeneous populations. Proc. nat. Acad. Sci., Wash. 43, 222.

Neumann, J. von (1951). Cerebral Mechanisms in Behaviour. (Cited by Haldane, New Biol. 16, 12.)

Novick, A. (1955). Growth of bacteria. Annu. Rev. Microbiol. 9, 97.

Novick, A. \& SziLARD, L. (1950). Experiments with the chemostat on spontaneous mutations of bacteria. Proc. nat. Acad. Sci., Wash. 36, 708.

Oparin, A. I. (1938). The Origin of Life (trans. Morgulis). New York: Macmillan.

Oparin, A. I. (1957). The Origin of Life on the Earth (3rd ed.) (trans. A. Synge). Edinburgh and London: Oliver and Boyd.

Penrose, L. S. \& Penrose, R. (1957). A self-reproducing analogue. Nature, Lond., 179, 1183.

Perret, C. J. (1956). The Technique of Cultivating Bacteria at Constant Population Density and its application to Studies of Adaptation. Ph.D. Thesis, University of London.

Perret, C. J. (1957). An apparatus for the continuous culture of bacteria at constant population density. J. gen. Microbiol. 16, 250.

Pirre, N. W. (1937). The meaninglessness of the terms life and living. In Perspectives in Biochemistry. Cambridge University Press.

PIrIe, N. W. (1953). Ideas and assumptions about the origin of life. Discovery, 14, 238.

PowLck, M. R. (1952). Penicillinase adaptation in Bacillus cereus: an analysis of three phases in the response of logarithmically growing cultures to induction of penicillinase formation by penicillin. Brit. J. exp. Path. 33, 587 .

PoLLock, M. R. (1953). Stages in enzyme adaptation. Symp. Soc. gen. Microbiol. 3, 150.

Reiner, J. M. \& Spiegelman, S. (1944). The energetics of transient and steady states with special reference to biological systems. J. phys. Chem. 49, 81 .

Schrödinger, E. (1944). What is Life? Cambridge University Press.

Spicer, C. (1955). The theory of bacterial constant growth apparatus. Biometrics, 11, 225.

Spiegrlman, S. \& Reiner, J. M. (1945). Maxima in rate-concentration curves and their relation to the structural aspects of cellular metabolism. J. gen. Physiol. 28, 385.

Volterra, V. (1931). Leçons sur la théorie mathématique de la lutte pour la vie. Paris: Gauthier-Villars. 